\title{
Thermal Hadron Production by QCD Hawking Radiation
}

\author{
Helmut Satz ${ }^{\dagger}$ \\ University of Bielefeld \\ E-mail: satz@physik.uni-bielefeld.de
}

The QCD counterpart of Hawking radiation from black holes leads to thermal hadron production in high energy collisions, from $e^{+} e^{-}$annihilation to heavy ion interactions. This hadronic radiation is formed by tunnelling through the confinement event horizon and is emitted at a universal temperature $T \simeq(\sigma / 2 \pi)^{1 / 2}$, where $\sigma$ denotes the string tension. Since the event horizon does not allow information transfer, the radiation is thermal "at birth"

Critical Point and Onset of Deconfinement

July 3-6 2006

Florence, Italy

\footnotetext{
*Speaker.

†Preliminary report on joint work with Paolo Castorina and Dmitri Kharzeev.
} 


\section{Introduction}

Quantum chromodynamics, through colour confinement, restricts quarks and gluons to move in a limited region of space. In a black hole, matter and light are confined by gravitation to remain within a restricted region of space. This similarity was noted quite soon after the advent of QCD, and it was suggested that hadrons were the analogue of black holes in strong interaction physics $[1,2,3]$. In the case of gravitational black holes, the isolation of the system is not quite absolute: in the strong field at the outer edge, quantum excitation can lead to the emission of Hawking radiation into the physical vacuum [4]. Since no information transfer between the inside and the outside of the black hole is allowed, this radiation must give equal a priori weights to all possible states on the outside, and hence it is thermal at the point of formation. In this report, based on the work of ref. [5], we want to show that high energy collisions in strong interaction physics produce a self-similar cascade of "white holes" (colourless from the outside, but coloured inside), and that the Hawking radiation arising at the confinement horizon of these provides the thermal behaviour observed quite universally in all soft hadron production $[6,7]$.

We begin by recalling briefly the relevant basic aspects of black hole physics and indicate some first relations to strong interactions. We then turn to high energy $e^{+} e^{-}$annihilation as the simplest hadron production process and study the effect of quark-antiquark pair excitation and string breaking. Next we review some features of charged and of rotating black holes; these allow us to generalize our scenario is to hadronic collisions at finite baryon density as well as to non-central interactions. and nuclear collisions. Finally, we comment on stochastic vs. kinetic thermalization.

\section{Black Holes and Hawking Radiation}

A black hole is the final stage of a neutron star after gravitational collapse [8]. It has a mass $M$ concentrated in such a small volume that the resulting gravitational field confines all matter and even photons to remain inside the event horizon $R$ of the system: no causal connection with the outside is possible. As a consequence, black holes have three (and only three) observable properties: mass $M$, charge $Q$ and angular momentum $J$. This section will address mainly black holes with $Q=J=0$; we shall come back to the more general properties in section 4 .

Classically, a black hole would persist forever and remain forever invisible. On a quantum level, however, its constituents (photons, leptons and hadrons) have a non-vanishing chance to escape by tunnelling through the barrier presented by the event horizon. The resulting Hawking radiation [4] cannot convey any information about the internal state of the black hole; it must be therefore be thermal, and it was shown that for a non-rotating black hole of vanishing charge (denoted as Schwarzschild black hole), the corresponding radiation temperature is

$$
T_{B H}=\frac{1}{8 \pi G M}
$$

where $G \simeq 6.7 \times 10^{-39} \mathrm{GeV}^{-2}$ is the gravitational constant, in units with $\hbar=c=1$. This temperature is inversely proportional to the mass of the black hole, and since the radiation reduces the mass, the radiation temperature will change with time. For black holes of stellar size, however, for $M \geq 2-3 M_{\odot}$, where $M_{\odot}$ denotes the solar mass, one finds $T_{B H} \lesssim 2 \times 10^{-8}{ }^{\circ} \mathrm{K}$. This is many 
orders of magnitude below the $2.7{ }^{\circ} \mathrm{K}$ cosmic microwave background, and hence not detectable; in fact, such black holes absorb more than they radiate and thus grow rather than decrease in mass. The search for cosmic Hawking radiation therefore considers the possible existence of primordial black holes of very much sub-stellar mass and size, formed very shortly after the big bang.

In general relativity, the event horizon arises as consequence of the Schwarzschild metric and its generalizations to $Q \neq 0, J \neq 0$. The occurrence and role of the event horizon for thermal radiation was subsequently generalized by Unruh [9]. A system undergoing uniform acceleration $a$ relative to a stationary observer eventually reaches a classical turning point and thus encounters an event horizon. Let us recall the resulting hyperbolic motion [10]. A point mass $m$ subject to a constant force $F$ satisfies the equation of motion

$$
\frac{d}{d t} \frac{m v}{\sqrt{1-v^{2}}}=F
$$

where $v(t)=d x / d t$ is the velocity, normalized to the speed of light $c=1$. This equation is solved by the parametric form

$$
x=\frac{1}{a} \cosh a \tau \quad t=\frac{1}{a} \sinh a \tau,
$$

where $a=F / m$ denotes the acceleration in the instantaneous rest frame of $m$, and $\tau$ the proper time, with $d \tau=\sqrt{1-v^{2}} d t$.

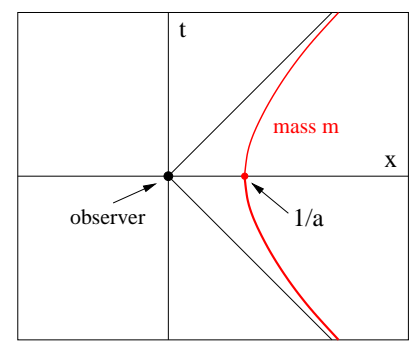

Figure 1: Hyperbolic motion

The resulting world line is shown in Fig. 1. It corresponds to the mass $m$ coming from $x=\infty$ at $t=-\infty$ at with a velocity arbitrarily close to that of light, decelerating uniformly until it comes to rest at the classsical turning point $x_{H}=-(1 / a)$. It then accelerates again and returns to $x=\infty$ at $t=\infty$, approaching the speed of light. For given $a, x_{H}$ is thus the event horizon beyond which $m$ classically cannot pass. The only possible signal beyond this point is thermal quantum radiation of temperature $[4,9,11,12]$

$$
T_{U}=\frac{a}{2 \pi}
$$

In the case of gravity, we have the force

$$
F=m a=G \frac{m M}{R^{2}},
$$

on a probe of mass $m$. From the dependence of the gravitational metric on black hole mass and radius, we obtain

$$
R_{g}=2 G M
$$


for the Schwarzschild radius of the black hole, leading to $a=1 /(4 G M)$ for the acceleration at the event horizon and thus back to eq. (2.1). We also see why the temperature of Hawking radiation decreases with increasing black hole mass: since $R_{g}$ grows linearly with $M$, increasing the black hole mass decreases its energy density and hence the Hawking temperature $T_{B H}$.

It is instructive to consider the Schwarzschild radius of a typical hadron, assuming a mass $m \sim 1 \mathrm{GeV}$ :

$$
R_{g}^{\text {had }} \simeq 1.3 \times 10^{-38} \mathrm{GeV}^{-1} \simeq 2.7 \times 10^{-39} \mathrm{fm} .
$$

To become a gravitational black hole, the mass of the hadron would thus have to be compressed into a volume more than $10^{100}$ times smaller than its actual volume, with a radius of about $1 \mathrm{fm}$. On the other hand, if instead we increase the interaction strength from gravitation to strong interaction [1], we gain in the resulting "strong" Schwarzschild radius $R_{s}^{\text {had }}$ a factor

$$
\frac{\alpha_{s}}{G m^{2}},
$$

where $\alpha_{s}$ is the dimensionless strong coupling and $G m^{2}$ the corresponding dimensionless gravitational coupling for the case in question. This leads to

$$
R_{s}^{h a d} \simeq \frac{2 \alpha_{s}}{m}
$$

which for $\alpha_{s} \simeq 2.5$ gives $R_{s}^{\text {had }}=1 \mathrm{fm}$. In other words, the confinement radius of a hadron is about the size of its "strong" Schwarzschild radius, so that we could consider quark confinement as the strong interaction version of the gravitational confinement in black holes [1, 2].

\section{Pair Production and String Breaking}

In the previous section, we had considered constituents of a black hole undergoing accelerated motion in classical space-time. In this section, we shall first address the modifications which arise if the underlying space-time manifold is specified by quantum field theory, so that in the presence of a strong field the vacuum becomes unstable under pair production. Next we turn to the specific additional features which come in when the basic constituents are subject to colour confinement and can only exist in colour neutral bound states.

As basic starting point, we consider two-jet $e^{+} e^{-}$annihilation at cms energy $\sqrt{s}$,

$$
e^{+} e^{-} \rightarrow \gamma \rightarrow q^{-} q \rightarrow \text { hadrons. }
$$

The initially produced $q^{-} q$ pair flies apart, subject to the constant confining force given by the string tension $\sigma$; this results in hyperbolic motion $[13,14,15]$ of the type discussed in the previous section. At $t=0$, the $q$ and ${ }^{-} q$ separate with an initial velocity $\forall=p / \sqrt{p^{2}+m^{2}}$, where $p \simeq \sqrt{s} / 2$ is the momentum of the primary constituents in the overall $\mathrm{cms}$ and $m$ the effective quark mass. We now have to solve Eq. (2.2) with this situation as boundary condition; the force

$$
F=\sigma
$$

is given by the string tension $\sigma$ binding the $q^{-} q$ system. The solution is

$$
\tilde{x}=\left[1-\sqrt{1-v_{0} \tilde{t}+\tilde{t}^{2}}\right]
$$


with $\tilde{x}=x / x_{0}$ and $\tilde{t}=t / x_{0}$; here the scale factor

$$
x_{0}=\frac{m}{\sigma} \frac{1}{\sqrt{1-v_{0}^{2}}}=\frac{1}{a} \gamma
$$

is the inverse of the acceleration $a$ measured in the overall $\mathrm{cms}$. The velocity becomes

$$
v(t)=\frac{d x}{d t}=\frac{\left(v_{0} / 2\right)-\tilde{t}}{\sqrt{1-v_{0} \tilde{t}+\tilde{t}^{2}}}
$$

it vanishes for

$$
\tilde{t}^{*}=\frac{v_{0}}{2} \Rightarrow t^{*}=\frac{v_{0}}{2} \frac{m}{\sigma} \gamma
$$

thus defining

$$
x\left(t^{*}\right)=\frac{m}{\sigma} \gamma\left(1-\sqrt{1-\left(v_{0}^{2} / 4\right)}\right) \simeq \frac{\sqrt{s}}{2 \sigma}
$$

as classical turning point and hence as the classical event horizon measured in the overall $\mathrm{cms}$ (see Fig. 2).

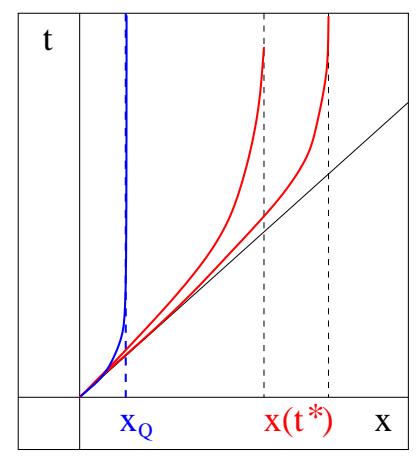

Figure 2: Classical and quantum horizons in $q \bar{q}$ separation

Eq. (3.7) allows the $q$ and the ${ }^{-} q$ to separate arbitrarily far, provided the pair has enough initial energy; this clearly violates colour confinement. Our mistake was to consider the $q^{-} q$ system in a classical vacuum; in quantum field theory, the vacuum itself contains virtual $q^{-} q$ pairs, and hence it is not possible to increase the potential energy of a given $q^{-} q$ state beyond the threshold value necessary to bring such a $q^{-} q$ pair on-shell. In QED, the corresponding phenomenon was addressed by Schwinger [16], who showed that in the presence of a constant electric field of strength $\mathscr{E}$ the probability of producing an electron-positron pair is given by

$$
P(M, \mathscr{E}) \sim \exp \left\{-\pi m_{e}^{2} / e \mathscr{E}\right\}
$$

with $m_{e}$ denoting the electron mass and $e$ denoting the electric charge. In QCD, we expect a similar effect when the string tension exceeds the pair production limit, i.e., when

$$
\sigma x>2 m
$$

where $m$ specifies the effective quark mass. Beyond this point, any further stretching of the string is expected to produce a $q^{-} q$ pair with the probability

$$
P(M, \sigma) \sim \exp \left\{-\pi m^{2} / \sigma\right\},
$$


with the string tension $\sigma$ replacing the electric field strength $e \mathscr{E}$. This string breaking acts like a quantum event horizon $x_{q}=2 \mathrm{~m} / \sigma$, which becomes operative long before the classical turning point is ever reached (see Fig. 2). Moreover, the resulting allowed separation distance for our $q^{-} q$ pair, the colour confinement radius $x_{Q}$, does not depend on the initial energy of the primary quarks.

There are some important differences between QCD and QED. In case of the latter, the initial electric charges which lead to the field $\mathscr{E}$ can exist independently in the physical vacuum, and the produced pair can be simply ionized into an $e^{+}$and an $e^{-}$. In contrast, neither the primary quark nor the constituents of the $q^{-} q$ pair have an independent existence, so that in string breaking colour neutrality must be preserved. As a result, the Hawking radiation in QCD must consist of $q^{-} q$ pairs, and these can be produced in an infinite number of different excitation states of increasing mass and degeneracy. Moreover, the $q^{-} q$ pair spectrum is itself determined by the strength $\sigma$ of the field, in contrast to the exponent $m_{e}^{2} / \mathscr{E}$ in eq. (3.8), where the value of $\mathscr{E}$ has no relation to the electron mass $m_{e}$.

Hadron production in $e^{+} e^{-}$annihilation is believed to proceed in the form of a self-similar cascade $[17,18]$. Initially, we have the separating primary $q^{-} q$ pair,

$$
\gamma \rightarrow\left[q^{-} q\right]
$$

where the square brackets indicate colour neutrality. When the energy of the resulting color flux tube becomes large enough, a further pair $q_{1}{ }^{-} q$ is excited from the vacuum,

$$
\left.\gamma \rightarrow\left[q^{-}{ }^{-} q_{1}\right]^{-} q\right] .
$$

Although the new pair is at rest in the overall $\mathrm{cms}$, each of its constituents has a transverse momentum $k_{T}$ determined, through the uncertainty relation, by the transverse dimension $r_{T}$ of the flux tube. The slow ${ }^{-} q$ now screens the fast primary $q$ from its original partner ${ }^{-} q$, with an analoguous effect for the $q_{1}$ and the primary antiquark. To estimate the $q^{-} q$ separation distance at the point of pair production, we recall that the basic thickness of the flux tube connecting the $q^{-} q$ pair is in string theory given by [19]

$$
r_{T}=\sqrt{\frac{2}{\pi \sigma}}
$$

higher excitations lead to a greater thickness and eventually to a divergence (the "roughening" transition). From the uncertainty relation we then have

$$
k_{T} \simeq \sqrt{\frac{\pi \sigma}{2}} .
$$

With this transverse energy is included in eq. (3.9), we obtain for the pair production separation $x_{Q}$

$$
\sigma x_{q}=2 \sqrt{m^{2}+k_{T}^{2}} \Rightarrow x_{q} \simeq \frac{2}{\sigma} \sqrt{m^{2}+(\pi \sigma / 2)} \simeq \sqrt{\frac{2 \pi}{\sigma}} \simeq 1.1 \mathrm{fm}
$$

with $\sigma=0.2 \mathrm{GeV}^{2}$ and $m^{2} \ll \sigma$.

Once the new pair is present, we have a colour-neutral system $q^{-} q q_{1}{ }^{-} q$; but since there is a sequence of connecting string potentials $q^{-} q,{ }^{-} q q_{1}$ and $q_{1}{ }^{-} q$, the primary string is not yet broken. To achieve that, the binding of the new pair has to be overcome, i.e., the $q_{1}$ has to tunnel through the 
barrier of the confining potential provided by ${ }^{-} q$, and vice versa. Now the $q$ excerts a longitudinal force on the ${ }^{-} q$, the ${ }^{-} q$ on the $q$, resulting in a longitudinal acceleration and ordering of $q_{1}$ and ${ }^{-} q$. When (see Fig. 3)

$$
\sigma x\left(q_{1}^{-} q\right)=2 \sqrt{m^{2}+k_{T}^{2}}
$$

the ${ }^{-} q$ reaches its $q_{1}{ }^{-} q$ horizon; on the other hand, when

$$
\sigma x\left(q^{-} q\right)=2 \sqrt{m^{2}+k_{T}^{2}},
$$

the new flux tube $q^{-} q$ reaches the energy needed to produce a further pair $q_{2}{ }^{-} q$. The ${ }^{-} q$ screens the primary $q$ from the $q_{1}$ and forms a new flux tube $q^{-} q$. At this point, the original string is broken, and the remaining pair ${ }^{-} q q_{2}$ form a colour neutral bound state which is emitted as Hawking radiation in the form of hadrons, with the relative weights of the different states governed by the corresponding Unruh temperature. The resulting pattern is schematically illustrated in Fig. 3.

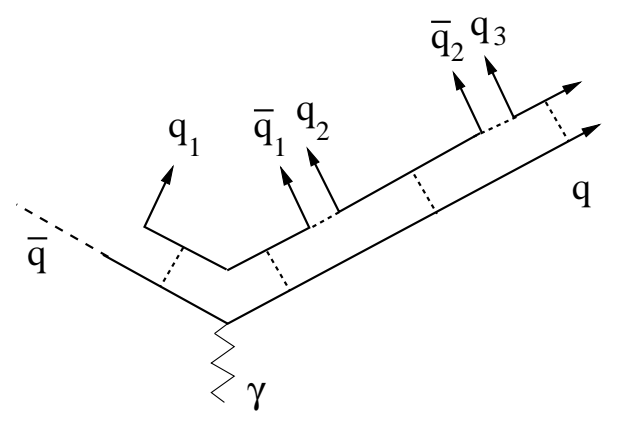

Figure 3: String breaking through $q \bar{q}$ pair production

To determine the temperature of the hadronic Hawking radiation, we return to the original pair excitation process. To produce a quark of momentum $k_{T}$, we have to bring it on-shell and change its velocity from zero to $v=k_{T} /\left(m^{2}+k_{T}^{2}\right)^{1 / 2} \simeq 1$. This has to be achieved in the time of the fluctuation determined by the virtuality of the pair, $\Delta \tau=1 / \Delta E \simeq 1 / 2 k_{T}$. The resulting acceleration thus becomes

$$
a=\frac{\Delta v}{\Delta \tau} \simeq 2 k_{T} \simeq \sqrt{2 \pi \sigma} \simeq 1.1 \mathrm{GeV}
$$

which leads to

$$
T_{q}=\frac{a}{2 \pi} \simeq \sqrt{\frac{\sigma}{2 \pi}} \simeq 180 \mathrm{MeV}
$$

for the hadronic Unruh temperature. It governs the momentum distribution and the relative species abundances of the emitted hadrons.

A given step in the evolution of the hadronization cascade of a primary quark or antiquark produced in $e^{+} e^{-}$annihilation thus involves several distinct phenomena. The color field created by the separating $q$ and ${ }^{-} q$ produces a further pair $q^{-} q$ and then provides an acceleration of the $q_{1}$, increasing its longitudinal momentum. When it reaches the $q_{1}{ }^{-} q$ confinement horizon, still another pair $q_{2}{ }^{-} q$ is excited; the state ${ }^{-} q q_{2}$ is emitted as a hadron, the ${ }^{-} q$ forms together with the primary $q$ a new flux tube. This pattern thus step by step increases the longitudinal momentum of the "accompanying" $q$ as well as of the emitted hadron. This, together with the energy of the 
produced pairs, causes a corresponding deceleration of the primary quarks $q$ and ${ }^{-} q$, in order to maintain overall energy conservation. In Fig. 4, we show the world lines given by the acceleration ${ }^{-} q \rightarrow{ }^{-} q_{+1}\left(q_{i} \rightarrow q_{i+1}\right)$ and that of formation threshold of the hadrons ${ }^{-} q q_{i+1}$ and the corresponding antiparticles.

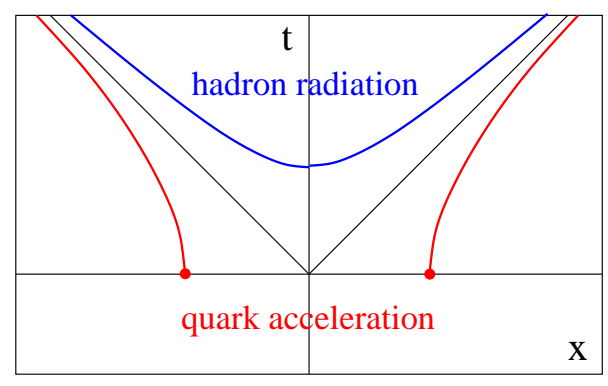

Figure 4: Quark acceleration and hadronization world lines

The energy loss and deceleration of the primary quark $q$ in this self-similar cascade, together with the acceleration of the accompanying partner ${ }^{-} q$ from the successive pairs brings $q$ and ${ }^{-} q$ closer and closer to each other in momentum, from an initial separation $q^{-} q$ of $\sqrt{s} / 2$, until they finally are combined into a hadron and the cascade is ended. The resulting pattern is shown in Fig. 5 .

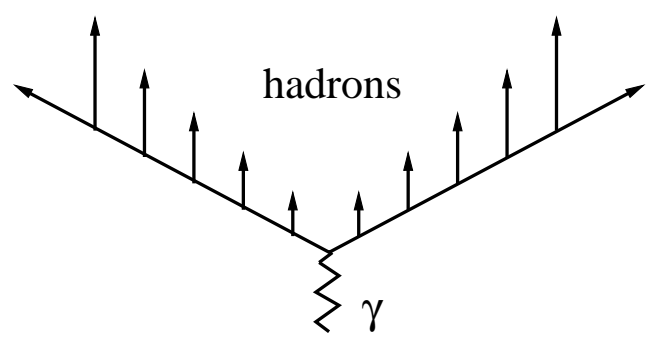

Figure 5: Hadronization in $e^{+} e^{-}$annihilation

The number of emitted hadrons, the multiplicity $v(s)$, follows quite naturally from the picture presented here. The classical string length, in the absence of quantum pair formation, is given by the classical turning point determined in eq. (3.7). The thickness of a flux tube of such an "overstretched" string is known [19]; instead of eq. (3.13) we have

$$
R_{T}^{2}=\frac{2}{\pi \sigma} \sum_{k=0}^{K} \frac{1}{2 k+1} \simeq \frac{2}{\pi \sigma} \ln 2 K
$$

where $K$ is the string length in units of an intrinsic string vibration measure. From eq. (3.7) we thus get

$$
R_{T}^{2}=\simeq \frac{2}{\pi \sigma} \ln \sqrt{s}
$$

for the flux tube thickness in the case of the classical string length. Because of pair production, this string breaks whenever it is stretched to the length $x_{q}$ given in eq. (3.15); its thickness $r_{T}$ at this point is given by eq. (3.13). The multiplicity can thus be estimated by the ratio of the corresponding 
classical to quantum transverse flux tube areas,

$$
v(s) \sim \frac{R_{T}^{2}}{r_{T}^{2}} \simeq \ln \sqrt{s}
$$

and is found to grow logarithmically with the $e^{+} e^{-}$annihilation energy, as is in fact observed experimentally.

Up to now, we have considered hadron production in $e^{+} e^{-}$annihilation, in which the virtual photon produces a confined coloured $q^{-} q$ pair as a "white hole". Turning now to hadron-hadron collisions, we note that here two incident white holes combine to form a new system of the same kind, as schematically illustrated in Fig. 6. Again the resulting string or strong colour field produces a sequence of $q^{-} q$ pairs of increasing cms momentum, leading to the well-known multiperipheral hadroproduction cascade shown in Fig. 7. For the further extension to nuclear collisions in the regime of parton saturation, see [20, 21, 22].

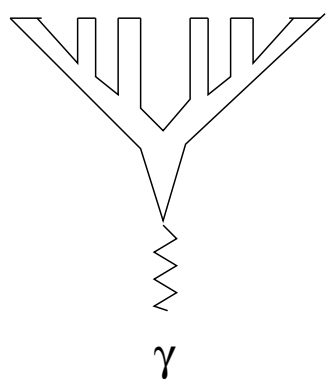

(a)

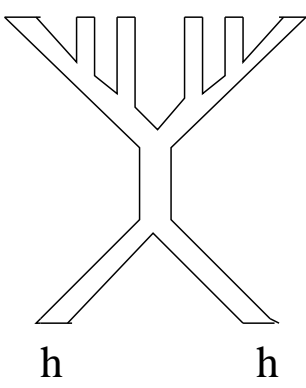

(b)

Figure 6: "White hole" structure in $e^{+} e^{-}$annihilation (a) and hadronic collisions (b)

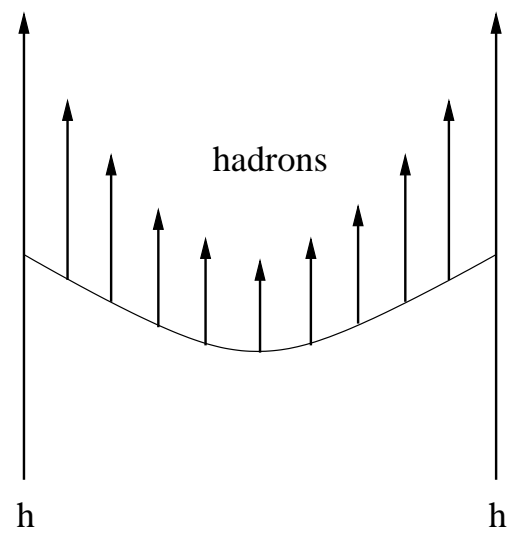

Figure 7: Hadronization in hadron-hadron collisions

In the case of hadronic, and even more so for heavy ion interactions, two new elements enter. The resulting system can now have an overall baryon number, from $\mathrm{B}=2$ in $p p$ to $B=400$ or more in heavy ion collisions. To take that into account, we need to consider the counterpart of charged black holes. Furthermore, in heavy ion collisions the resulting hadron production can be studied 
as function of centrality, and peripheral collisions will lead to an interaction region with an overall angular momentum. Hence we also need to consider rotating black holes. In the next section, we therefore summarize the relevant feature of black holes with $Q \neq 0, J \neq 0$.

\section{Charged and Rotating Black Holes}

As mentioned, an outside observer the only characteristics of a black hole are its mass $M$, its electric charge $Q$, and its spin or angular momentum $J$. Hence any further observables, such as the event horizon or the Hawking temperature, must be expressable in terms of these three quantities.

The event horizon of a black hole is created by the strong gravitational attraction, which leads to a diverging Schwarzschild metric at a certain value of the spatial extension $R$. Specifically, the invariant space-time length element $d s^{2}$ is at the equator given by

$$
d s^{2}=(1-2 G M / R) d t^{2}-\frac{1}{1-2 G M / R} d r^{2},
$$

with $r$ and $t$ for flat space and time coordinates; it is seen to diverge at the Schwarzschild radius $R_{S}=2 G M$. If the black hole has a net electric charge $Q$, the resulting Coulomb repulsion will oppose and hence weaken the gravitational attraction. As a result, the corresponding form (denoted as Reissner-Nordström metric) becomes

$$
d s^{2}=\left(1-2 G M / R+G Q / R^{2}\right) d t^{2}-\frac{1}{1-2 G M / R+G Q / R^{2}} d r^{2} .
$$

For this, the divergence leads to the smaller Reissner-Nordström radius

$$
R_{R N}=G M\left(1+\sqrt{1-Q^{2} / G M^{2}}\right),
$$

which reduces to the Schwarzschild radius $R_{S}$ for $Q=0$. The temperature of the Hawking radiation now becomes $[8,23]$

$$
T_{B H}(M, Q)=T_{B H}(M, 0)\left\{\frac{2 \sqrt{1-Q^{2} / G M^{2}}}{\left(1+\sqrt{1-Q^{2} / G M^{2}}\right)^{2}}\right\} ;
$$

its functional form is illustrated in Fig. 8. We note that with increasing charge, the Coulomb repulsion weakens the gravitational field at the event horizon and hence decreases the temperature of the corresponding quantum excitations. As $Q^{2} \rightarrow G M^{2}$, the gravitational force is fully compensated and the black hole is gone.

In a similar way, the effect of the angular momentum of a rotating black hole can be incorporated. It is now the centripetal force which counteracts the gravitational attraction and hence reduces its strength. The resulting Kerr metric must take into account that in this case the rotational symmetry is reduced to an axial symmetry, and with $\theta$ denoting the angle relative to the polar axis $\theta=0$, it is (at fixed longitude) given by

$$
d s^{2}=\left(1-\frac{2 G M R}{R^{2}+a^{2} \cos ^{2} \theta}\right) d t^{2}-\frac{R^{2}+a^{2} \cos ^{2} \theta}{R^{2}-2 G M R+a^{2}} d r^{2}-\left(R^{2}+a^{2} \cos ^{2} \theta\right) d \theta^{2} .
$$




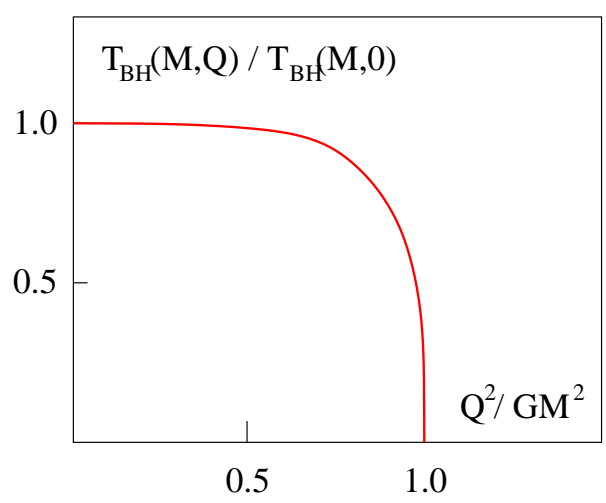

Figure 8: Radiation temperature for a charged black hole

The angular momentum of the black hole is here specified by the parameter $a=J / M$; for $a=0$, we again recover the Schwarzschild case. The general situation is now somewhat more complex, since eq. (4.5) leads to two different divergence points. The solution

$$
R_{K}=G M\left(1+\sqrt{1-a^{2} /(G M)^{2}}\right)
$$

defines the actual event horizon, corresponding to absolute confinement. But the resulting black hole is now embedded in a larger ellipsoid

$$
R_{E}=G M\left(1+\sqrt{1-\left[a^{2} /(G M)^{2}\right] \cos ^{2} \theta}\right),
$$

as illustrated in Fig. 9. The two surfaces touch at the poles, and the region between them is denoted as the ergosphere. Unlike the black hole proper, communication between the ergosphere and the outside world is possible. Any object in the ergosphere will, however, suffer from the rotational drag of the rotating black hole and thereby gain momentum. We shall return to this shortly; first, however, we note that the temperature of the Hawking radiation from a rotating black hole becomes

$$
T_{B H}(M, J)=T_{B H}(M, 0)\left\{\frac{2 \sqrt{1-a^{2} /(G M)^{2}}}{\left(1+\sqrt{1-a^{2} /(G M)^{2}}\right.}\right\} .
$$

For a non-rotating black hole, with $a=0$, this also reduces to the Hawking temperature for the Schwarzschild case.

To illustrate the effect of the ergosphere, imagine radiation from a Schwarzschild black hole emitted radially outward from the event horizon. In the case of a Kerr black hole, such an emission is possible only along the polar axis; for all other values of $\theta$, the momentum of the emitted radiation (even light) will increase due to the rotational drag in the ergosphere. This effect ceases only once the radiation leaves the ergosphere. Since the amount of drag depends on $\theta$, the momentum of the radiation emitted from a rotating black hole, as measured at large distances, will depend on the latitude at which it is emitted and increase from pole to equator.

Finally, for completeness, we note that for black holes with both spin and charge (denoted as Kerr-Newman), the event horizon is given by

$$
R_{K N}=G M\left(1+\sqrt{1-\left[Q^{2} / G M^{2}\right]-\left[a^{2} /(G M)^{2}\right]}\right),
$$




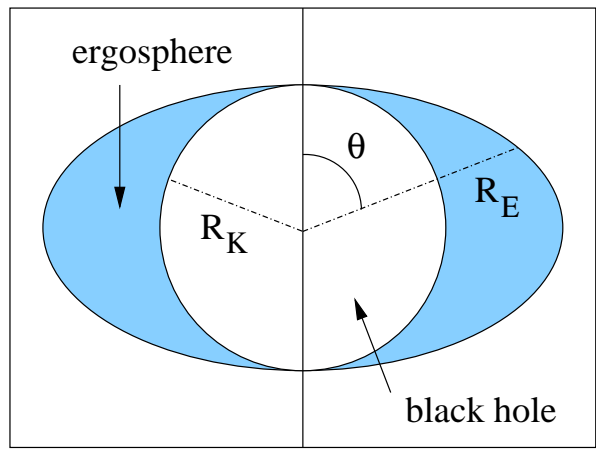

Figure 9: Geometry of a rotating black hole

and the radiation temperature becomes $[8,23]$

$$
T_{B H}(M, Q, J)=T_{B H}(M, 0,0)\left\{\frac{4 \sqrt{1-\left(G Q^{2}+a^{2}\right) /(G M)^{2}}}{\left(1+\sqrt{1-\left(G Q^{2}+a^{2}\right) /(G M)^{2}}\right)^{2}+a^{2} /(G M)^{2}}\right\} .
$$

The decrease of $T_{B H}$ for $Q \neq 0, J \neq 0$ expresses the fact that both the Coulomb repulsion and the rotational force counteract the gravitational attraction, and if they win, the black hole is dissolved.

\section{Vacuum Pressure and Baryon Density}

We now want to consider the extension of charged black hole physics to colour confinement in the case of systems with a net baryon number. In eq. (4.4) we had seen that the reduction of the gravitational attraction by Coulomb repulsion in a charged black hole in turn reduces the temperature of Hawking radiation. The crucial quantity here is the ratio $Q^{2} / G M^{2}$ of the overall Coulomb energy, $Q^{2} / R$, to the overall gravitational energy, $G M^{2} / R$. Equivalently, $Q^{2} / G M^{2}=$ $P_{Q} / P_{G}$ measures the ratio of inward gravitational pressure $P_{G}$ at the event horizon to the repulsive outward Coulomb pressure $P_{Q}$.

In QCD, we have a "white" hole containing coloured quarks, confined by chromodynamic forces or, equivalently, by the pressure $B$ of the physical vacuum. If the system has a non-vanishing overall baryon number, there will be a Fermi repulsion between the corresponding quarks, and this repulsion will provide a pressure $P(\mu)$ acting against $B$, with $\mu$ denoting the corresponding quark baryochemical potential. We thus expect a similar reduction of the hadronization temperature as function of $\mu$. To quantify this, we have to obtain the reduction of the chromodynamic force field, such as the string tension $\sigma$, due to baryonic repulsion. As a first estimate, we start from the simplest picture of colour confinement and consider is an ideal gas of massless quarks and gluons, held together by the vacuum pressure $B$. At fixed temperature $T$ and quark baryochemical potential $\mu$, the overall pressure of such a system is given by

$$
P=\left(\frac{\pi^{2}}{90}\left[d_{b}+\frac{7}{4} d_{f}\right]\right) T^{4}+\left(\frac{d_{f}}{12}\right) \mu^{2} T^{2}+\left(\frac{d_{f}}{24 \pi^{2}}\right) \mu^{4}-B
$$

where $d_{b}$ and $d_{f}$ denote the degrees of freedom of gluons and quarks, respectively; for colour $S U(3)$ and two quark flavours, $d_{b}=16$ and $d_{f}=12$. When the inward vacuum pressure just balances the 
combined outward kinetic motion of quarks and gluons and the fermi repulsion of the quarks, we have $P=0$. For $\mu=0$, this gives

$$
T_{0}=\left(\frac{90}{37 \pi^{2}}\right)^{1 / 4} B^{1 / 4} \simeq 0.70 B^{1 / 4}
$$

On the other hand, for $T=0$, when the Fermi repulsion of the quarks alone balances the vacuum pressure, $\mu$ becomes

$$
\mu_{0}=\left(2 \pi^{2} B\right)^{1 / 4}=\pi\left(\frac{37}{45}\right)^{1 / 4} T_{0} \simeq 3 T_{0} .
$$

With a hadronization temperature $T_{0} \simeq 0.175 \mathrm{GeV}$, this leads to a baryochemical potential $\mu_{b}=$ $3 \mu \simeq 1.6 \mathrm{GeV}$. In the intermediate region, where both $T$ and $\mu$ are finite, we want to compare the effect of the Fermi repulsion to the vacuum pressure through the Hawking-Unruh form, i.e., we replace $Q^{2} / M^{2}$ in eq. (4.4) by $\mu / \mu_{0}$, giving

$$
T(\mu) / T_{0}=\frac{\sqrt{1-\left(\mu / \mu_{0}\right)^{4}}}{\left(1+\sqrt{1-\left(\mu / \mu_{0}\right)^{4}}\right)^{2}} .
$$

The resulting behaviour of $T(\mu)$ is shown in Fig. 10 .

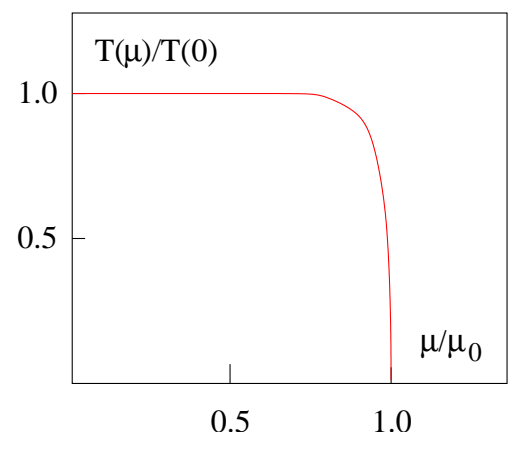

Figure 10: Hadronization temperature as function of the baryochemical potential

Instead of using the hadronization temperature as scale, the vacuum pressure can be determined in terms of the gluon condensate $\left\langle G_{\mu \nu}^{2}\right\rangle[24,25]$,

$$
B=\frac{33-2 N_{f}}{384 \pi^{2}}\left\langle G_{\mu \nu}^{2}\right\rangle .
$$

Numerical studies [24] lead for two flavours to $B^{1 / 4} \simeq 0.21-0.25 \mathrm{GeV}$; from this we get both $T_{0} \simeq 0.150-0.175 \mathrm{GeV}$ and $\mu_{0} \simeq 1.3-1.6 \mathrm{GeV}$.

Clearly this approach is overly simplistic, since it reduces the effect of the additional quarks to only their Fermi repulsion. A more general way of addressing the problem would be to introduce an effective $\mu$-dependence of the string tension. The presence of further quarks will lead to a screening-like reduction of the force between a given $Q \bar{Q}$ pair, and hence a screened string tension, with a $\mu$-dependent screening mass as obtained in finite density lattice studies, might provide a more realistic approach to the $\mu$ dependence of the Hawking-Unruh hadronization temperature. 


\section{Angular Momentum and Non-Central Collisions}

The dependence of Hawking radiation on the angular momentum of the emitting system introduces a particularly interesting aspect for the "white hole evaporation" we have been considering. Consider a nucleus-nucleus collision at non-zero impact parameter $b$. If the interaction is of collective nature, the resulting system will have some angular momentum orthogonal to the reaction plane (see Fig. 11). For central collisions, this will not be the case, nor for extremely peripheral ones, where one expects essentially just individual nucleon-nucleon collisions without collective effects.

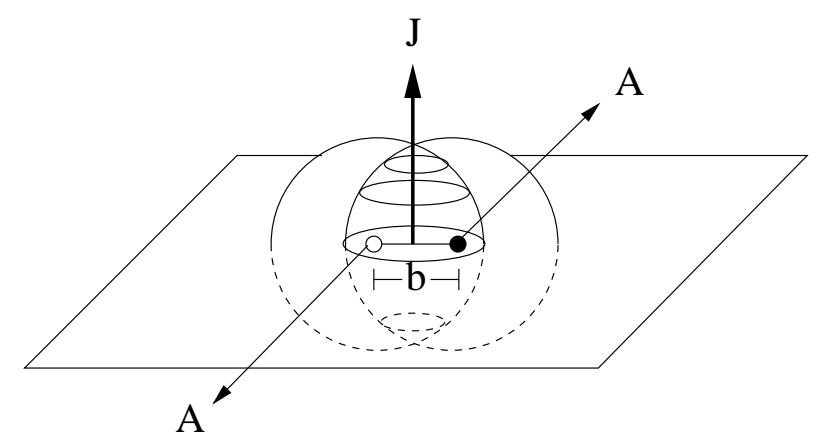

Figure 11: Rotating interaction region in non-central $A A$ collision

If it possible to consider a kinematic region in which the interacting system does have an overall spin, then the resulting Hawking radiation temperature should be correspondingly reduced, as seen in eq. (4.8). The effect is not so easily quantified, but simply a reduction of the hadronization temperature for non-central collisions would quite indicative. Such a reduction could appear only in the temperature determined by the relative abundances, since, as we shall see shortly, the transverse momentum spectra should show modifications due to the role of the ergosphere. The hadronization temperature determined from a resonance gas study is thus expected to start for central collisions with the value (3.19) or, at finite $\mu$ with the corresponding $T_{H}(\mu)$, then decrease with the onset of white hole rotation for non-central collisions, and finally increase again as collective nuclear effects go away and we recover elementary nucleon-nucleon collisions. The effect is schematically illustrated in Fig. 12

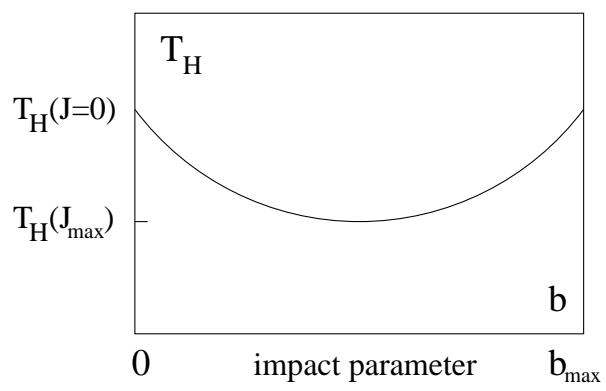

Figure 12: Schematic view of temperature variation with centrality

We next turn to the momentum spectrum of the Hawking radiation emitted from a rotating white hole. As discussed in section 4 , such radiation will exhibit an azimuthal asymmetry due 
to the presence of the ergosphere, which by its rotation will affect the momentum spectrum of any passing object. At the event horizon, the momentum of all radiation is determined by the corresponding Hawking temperature (4.8); but the passage of the ergosphere adds rotational motion to the emerging radiation and hence increases its momentum. As a result, only radiation emitted directly along the polar axis will have momenta as specified by the Hawking temperature; with increasing latitude $\theta$ (see Fig. 13)a, the rotation will increase the radiation momentum up to a maximum value in the equatorial plane.

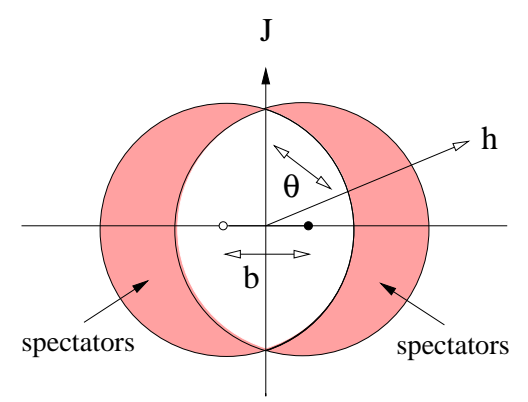

(a)

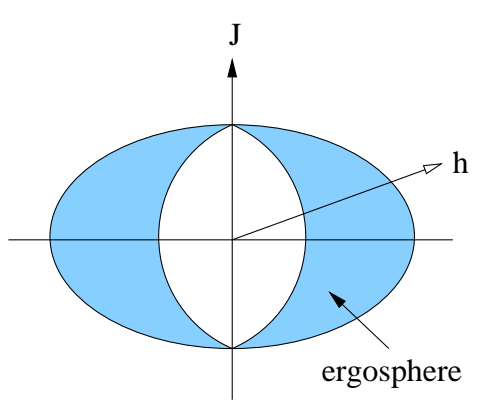

(b)

Figure 13: Transverse plane view of a non-central $A A$ collision

Hawking radiation from a rotating source thus leads for nuclear collisions quite naturally to what in hydrodynamic studies is denoted as elliptic flow. It is interesting to note that both scenarios involve collective effects: while in hydrodynamics, it is assumed that non-central collisions lead to an azimuthally anisotropic pressure gradient, we have here assumed that such collisions lead to an overall angular momentum of the emitting system.

\section{Stochastic vs. Kinetic Thermalization}

In statistical mechanics, a basic topic is the evolution of a system of many degrees of freedom from non-equilibrium to equilibrium. Starting from a non-equilibrium initial state of low entropy, the system is assumed to evolve as a function of time through collisions to a time-independent equlibrium state of maximum entropy. In other words, the system loses the information about its initial state through a sequence of collisions and thus becomes thermalized. In this sense, thermalization in heavy ion collisions was studied as the transition from an initial state of two colliding beams of "parallel" partons to a final state in which these partons have locally isostropic distributions. This "kinetic" thermalization requires a sufficient density of constituents, sufficiently large interaction cross sections, and a certain amount of time.

$>$ From such a point of view, the observation of thermal hadron production in high energy collisions in general, in $e^{+} e^{-}$and $p p$ interactions as well as in heavy ion collisions, is a puzzle: how could these systems ever "have reached" thermalization? Already Hagedorn had therefore concluded that the emitted hadrons were "born in equilibrium" [26].

Hawking radiation provides a stochastic rather than kinetic approach to equilibrium. The barrier to information transfer provided by the event horizon requires that the resulting radiation states 
excited from the vacuum are distributed according to maximum entropy, with a temperature determined by the strength of the "confining" field. The ensemble of all produced hadrons, averaged over all events, then leads to the same equilibrium distribution as obtained in hadronic matter by kinetic equilibration. In the case of a very high energy collision with a high average multiplicity already one event can provide such equilibrium; because of the interruption of information transfer at each of the successive quantum colour horizons, there is no phase relation between two successive production steps in a given event. The destruction of memory, which in kinetic equilibration is achieved through sufficiently many successive collisions, is here automatically provided by the tunnelling process.

So the thermal hadronic final state in high energy collisions is not reached through a kinetic process; it is rather provided by successively throwing dice.

\section{Conclusions}

We have shown that quantum tunnelling through the colour confinement horizon leads to thermal hadron production in the form of Hawking radiation. In particular, this implies:

- The radiation temperature $T_{q}$ is determined by the transverse extension of the colour flux tube, giving

$$
T_{q}=\sqrt{\frac{\sigma}{2 \pi}}
$$

in terms of the string tension $\sigma$.

- The multiplicity $v(s)$ of the produced hadrons is determined by the increase of the flux tube thickness with string length, leading to

$$
v(s) \simeq \ln \sqrt{s},
$$

where $\sqrt{s}$ denotes the collision energy.

- The temperature of Hawking radiation can in general depend on the charge and the angular momentum of the emitting system. The former here leads to the dependence of the hadronization temperature on baryon density. The latter results in elliptic flow and in a prediction of $T_{H}$ on the centrality of $A A$ collisions.

- In statistical QCD, thermal equilibrium is reached kinetically from an initial non-equilibrium state, with memory destruction through successive interactions of the constituents. In high energy collisions, tunnelling prohibits information transfer and hence leads to stochastic production, so that we have a thermal distribution from the outset.

\section{References}

[1] E. Recami and P. Castorina, Lett. Nuovo Cim. 15 (1976) 347.

[2] A. Salam and J. Strathdee, Phys. Rev. D18 (1978).

[3] A. F. Grillo and Y. Srivastava, Phys. Lett. B 85 (1979) 377. 
[4] S. W. Hawking, Comm. Math. Phys. 43 (1975) 199.

[5] P. Castorina, D. Kharzeev and H. Satz, in progress.

[6] R. Hagedorn, Nuovo Cim. Suppl. 3 (1965) 147; Nuovo Cim. A 56 (1968) 1027

[7] F. Becattini, Z. Phys. C69 (1996) $485\left(e^{+} e^{-}\right)$;

F. Becattini and U. Heinz, Z. Phys. C76 (1997) 268 ( $p p / p \bar{p})$;

J. Cleymans and H. Satz, Z. Phys. C57 (1993) 135 (heavy ions);

F. Becattini et al., Phys. Rev. C64 (2001) 024901 (heavy ions);

P. Braun-Munziger, K. Redlich and J. Stachel, (heavy ions).

[8] See e.g., Li Zhi Fang and R. Ruffini, Basic Concepts in Relativistic Astrophysics, World Scientific, Singapore 1983.

[9] W. G. Unruh, Phys. Rev. D14 (1976) 870.

[10] For a clear discussion and references to the original solutions by M. Born (1909) and A. Sommerfeld (1910),, see W. Pauli, Relativitätstheorie, in Enzyklopädie der mathematischen Wissenschaften, Teubner Verlag, Leipzig 1921; English version Theory of Relativity, Pergamon Press, 1958.

[11] T. D. Lee, Nucl. Phys. B264 (1986) 437.

[12] M. K. Parikh and F. Wilczek, Phys. Rev. Lett. 85 (2000) 5042.

[13] S. Barshay and W. Troost, Phys. Lett. 73B (1978) 437

[14] A. Hosoya, Progr. Theoret. Phys. 61 (1979) 280.

[15] M. Horibe, Progr. Theoret. Phys. 61 (1979) 661.

[16] J. Schwinger, Phys. Rev. 82 (1951) 664.

[17] J. D. Bjorken, Lecture Notes in Physics (Springer) 56 (1976) 93.

[18] A. Casher, H. Neuberger and S. Nussinov, Phys. Rev. D20 (1979) 179.

[19] M. Lüscher, G. Münster and P. Weisz, Nucl. Phys. B180 (1981) 1.

[20] D. Kharzeev and K. Tuchin, Nucl. Phys. A753 (2005) 316;

D. Kharzeev, hep-ph/0511354.

[21] D. Kharzeev, E. Levin and K. Tuchin, hep-ph/0602063.

[22] J. Dias de Deus and C. Pajares, hep-ph/0605148

[23] S. Iso, H. Umetsu and F. Wilczek, hep-th/0606018

[24] M. A. Shifman, A. I. Vainshtein and V. I. Zakharov, Nucl. Phys. B 147 (1979) 385.

[25] E. V. Shuryak, Phys. Rep. 61 (1980) 72.

[26] R. Hagedorn, Thermodynamics of Strong Interactions, CERN 71-12 (1971);

R. Stock, Phys. Lett. B 456 (1999) 277. 